\title{
Near-perfect tunneling and amplification of evanescent electromagnetic waves in a waveguide filled by a metamaterial: Theory and experiments
}

\author{
J. D. Baena,,${ }^{1, *}$ L. Jelinek, ${ }^{2}$ R. Marqués, ${ }^{1}$ and F. Medina ${ }^{1}$ \\ ${ }^{1}$ Departamento de Electrónica y Electromagnetismo, Universidad de Sevilla, 41012-Sevilla, Spain \\ ${ }^{2}$ Department of Electromagnetic Field, Czech Technical University, Prague, Czech Republic
}

(Received 29 November 2004; revised manuscript received 23 February 2005; published 10 August 2005)

\begin{abstract}
Amplification of evanescent electromagnetic waves by a metamaterial slab is still a controversial issue. Metallic waveguides operating in cutoff provide a unique physical environment to produce and study these waves because isolated evanescent modes can be excited in such environment. In this paper, the tunneling of electromagnetic waves through a rectangular waveguide partially filled by a metamaterial is analyzed. Enhanced tunneling due to the amplification of evanescent waves in the metamaterial filled waveguide section is demonstrated. Since evanescent modes in rectangular waveguides are the superposition of two plane waves, the aforementioned results also show the amplification and tunneling of evanescent plane waves by a metamaterial slab in free space.
\end{abstract}

DOI: $10.1103 /$ PhysRevB.72.075116

PACS number(s): 78.20.Ci, 41.20.Jb, 42.70.Qs, 78.20.Ek

\section{INTRODUCTION}

Amplification of evanescent electromagnetic waves in a left-handed ${ }^{1}$ slab is the physical phenomenon underlying the "perfect lens" effect recently described by Pendry. ${ }^{2}$ As reported in Ref. 2, a decaying electromagnetic mode impinging on the surface of a left-handed slab with $\epsilon=-\epsilon_{0}$ and $\mu=-\mu_{0}$ is turned to an amplifying wave inside the metamaterial. This amplification process can cancel the decay of evanescent waves in free space and restore the field amplitude at some plane behind the slab allowing for the aforementioned "perfect lens" effect. Although this amplification does not violate energy conservation since evanescent waves do not transport energy, ${ }^{2}$ this effect is actually unexpected in a passive device and was neither observed nor predicted before. Furthermore, this effect has been theoretically predicted in a pair of consecutive negative- $\epsilon$ and negative- $\mu$ slabs, ${ }^{3}$ and in a slab of a medium with indefinite tensor permittivity and/or permeability. ${ }^{4}$ Experimental observation of this phenomenon by means of subwavelength focusing experiments in a circuit analogous of these media has been recently reported.,6 More recently, it has been demonstrated that a system of two coupled planar sheets possessing surface mode resonances can amplify the evanescent field. ${ }^{7}$ Since it is possible to excite a single evanescent electromagnetic mode in a waveguide operating in cutoff, this environment constitutes a unique opportunity for the study of such effect, avoiding cumbersome discussions on the convergence of field integrals in free space configurations. ${ }^{8}$ Moreover, it is well known ${ }^{9}$ that the fundamental electromagnetic mode in a rectangular waveguide (see Fig. 1) is the superposition of two symmetrical plane waves with $k_{x}= \pm \sqrt{\pi / a}$. Thus, the results obtained in such environment also show the behavior of plane waves in free space. Therefore, we can conclude that the analysis of the amplification of evanescent waves through a rectangular waveguide filled by a metamaterial is an unique opportunity for the theoretical analysis and the experimental observation of the amplification of evanescent electromagnetic waves in these media.
As already mentioned, a single evanescent mode cannot carry energy. However, some energy flux between the emitter and the receiver must be present in any measurement of the aforementioned "perfect lens" effect. ${ }^{10}$ This means that a symmetrical set of modes with a decaying-amplifyingdecaying behavior in the backward direction (i.e., from the probe to the source) should be also excited in the receiver. The interference of this set of modes with the aforementioned modes excited at the input is what allows for energy transmission. In a waveguide configuration, the transmission of energy between the input and the output means that some tunneling through the in-cutoff waveguide sections must be present. It will be shown by theory and experiments that, under some circumstances, the amplitudes of the backward set of evanescent modes equal those of the forward one, producing a total transmission of power in the waveguide. The condition for this "perfect tunneling" will be shown to be in correspondence with the condition of amplification of evanescent modes when the output port (the receiver) is removed. Thus the reported "perfect tunneling" effect in waveguides is closely related to the aforementioned amplification of evanescent modes and "perfect lens" effects in lefthanded slabs. We feel that a similar "perfect tunneling" will take place in a symmetrical "perfect lens" experiment, when the emitter and the receiver are identical. Of course, losses will affect this perfect tunneling, reducing the amount of transmitted energy. However, as will be shown in the following, a still measurable tunneling enhancement can be measured in practical devices.

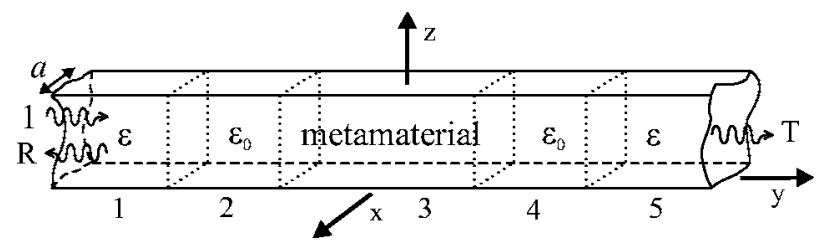

FIG. 1. A metamaterial filled waveguide sandwiched between two empty waveguides operating in cutoff, and two input/output waveguides above cutoff. The length of each waveguide sections is $l_{i}$. 


\section{THEORY}

Figure 1 shows an ideal experiment of perfect tunneling by amplification of evanescent waves in a rectangular waveguide section filled by a metamaterial. The three sections 2 , 3 , and 4 are in cutoff, so as they only support evanescent modes. An electromagnetic signal comes in and out of the device through two semi-infinite waveguide sections operating above cutoff (this can be achieved by simply filling these waveguides with a medium having $\epsilon>\epsilon_{0}$ ). The incident wave is the fundamental $T E_{10}$ mode and, since all the interfaces have the same cross section, no other modes are excited along the structure. Therefore the electric and magnetic fields have the form: ${ }^{9}$

$$
\begin{gathered}
\mathbf{E}_{ \pm}=\left(0,0, E_{z}^{0} \cos (\pi x / a)\right) \exp ( \pm i \beta y), \\
\mathbf{H}_{ \pm}=\left(H_{x}^{0} \cos (\pi x / a), H_{y}^{0} \sin (\pi x / a), 0\right) \exp ( \pm i \beta y),
\end{gathered}
$$

where $E_{z}^{0}, H_{x}^{0}$, and $H_{y}^{0}$ are functions of the frequency and the waveguide width, but not of position. The plus sign means that the wave flows from left to right (forward wave), and for minus sign in the opposite direction (backward wave). The propagation constant $\beta$ is a real number for the input and output sections, but it is imaginary for the three sections 2,3 , and 4 , being $\beta=i \alpha$, where $\alpha$ is the attenuation constant.

The transmission and reflection coefficients, $T$ and $R$, are obtained by simply cascading the scattering matrices for the fundamental mode in each waveguide section, ${ }^{9}$

$$
\left(\begin{array}{l}
1 \\
R
\end{array}\right)=\overline{\overline{\mathbf{Z}}}_{1,2} \cdot \overline{\overline{\mathbf{T}}}_{2} \cdot \overline{\overline{\mathbf{Z}}}_{2,3} \cdot \overline{\overline{\mathbf{T}}}_{3} \cdot \overline{\overline{\mathbf{Z}}}_{3,4} \cdot \overline{\mathbf{T}}_{4} \cdot \overline{\overline{\mathbf{Z}}}_{4,5}\left(\begin{array}{l}
T \\
0
\end{array}\right),
$$

where

$$
\begin{gathered}
\overline{\overline{\mathbf{Z}}}_{i, j}=\frac{1}{2 Z_{j}}\left(\begin{array}{cc}
Z_{j}+Z_{i}, & Z_{j}-Z_{i} \\
Z_{j}-Z_{i}, & Z_{j}+Z_{i}
\end{array}\right), \\
\overline{\mathbf{T}}_{i}=\left(\begin{array}{cc}
\exp \left(\alpha_{i} l_{i}\right), & 0 \\
0, & \exp \left(-\alpha_{i} l_{i}\right)
\end{array}\right),
\end{gathered}
$$

and $\alpha_{i}, Z_{i}$ are the attenuation constant and the impedance of the evanescent $T E_{10}$ mode in each waveguide section. If

$$
Z_{2}=Z_{3}=Z_{4} \text { and } l_{2} \alpha_{2}+l_{3} \alpha_{3}+l_{4} \alpha_{4}=0,
$$

it can be easily realized that $T=1$ and $R=0$, that is the amplitude of the incident evanescent wave is restored at the output, and the tunneling is perfect.

\section{A. Isotropic metamaterial case}

For isotropic media, ${ }^{9} \alpha_{i}=\sqrt{(\pi / a)^{2}-\omega^{2} \mu_{i} \epsilon_{i}}, Z_{i}=-i \omega \mu_{i} / \alpha_{i}$, and (6) reduces to the well known condition for the "perfect lens" effect, ${ }^{2}$

$$
\epsilon_{3}=-\epsilon_{0}, \quad \mu_{3}=-\mu_{0}, \quad \text { and } l_{2}+l_{4}=l_{3} .
$$

The physical interpretation of (6) and (7) is straightforward, since $\alpha_{3}$ should have the opposite sign to $\alpha_{2}$ and $\alpha_{4}$, any decaying evanescent mode generated at the input transition becomes an amplifying mode at the central waveguide sec-
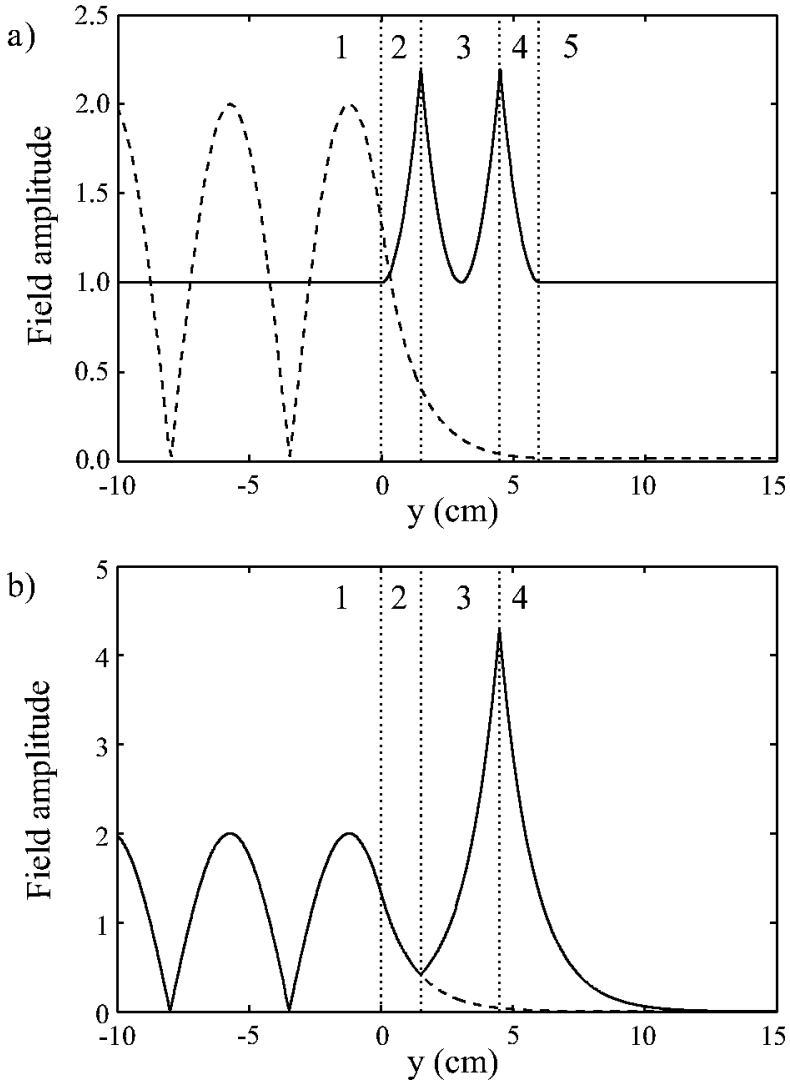

FIG. 2. Field amplitude distribution inside the structure of Fig. 1 , when section 3 is filled by an isotropic metamaterial with $\epsilon=-\epsilon_{0}, \mu=-\mu_{0}$. (a) Perfect tunneling when $l_{3}=2 l_{2}=2 l_{4}$. (b) Field amplitude when the last transition is eliminated $\left(l_{4} \rightarrow \infty\right)$. Dashed lines show the amplitude when section 3 is empty. The used geometrical dimensions are $a=24 \mathrm{~mm}, l_{2}=l_{4}=15 \mathrm{~mm}, l_{3}=30 \mathrm{~mm}$, and the operating frequency is $5 \mathrm{GHz}$. The dielectric constant of sections 1 and 5 is $\epsilon=2 \epsilon_{0}$.

tion (that filled with the metamaterial), becoming a new decaying in section 4 . Since the three sections are matched $\left(Z_{2}=Z_{3}=Z_{4}\right)$, no additional modes are generated at the 2-3 and 3-4 transitions. However, a similar set of modes (backward modes) is generated at the output port which allows for a nonzero flux of energy through the system (according to the tunneling theory, it is the interference between both sets of evanescent modes which allows for energy transmission). Due to the symmetry of the structure, the amplitude of both sets of modes must be equal. The resulting field distribution is summarized in Fig. 2(a) for the considered particular parameters. For comparison purposes, the field distribution is also shown in the figure when section 3 is empty. In order to show the relation with the amplification of evanescent modes in the purely reactive configuration, the field amplitude for a similar configuration but with $l_{4} \rightarrow \infty$ is shown in Fig. 2(b). In this case, since no transmission of energy is allowed, a single set of evanescent modes is present, and the amplitude of the field at the input port is restored at a distance $2 l_{3}$ from it.

Until now we have only considered a lossless metamaterial, but in a realistic model losses should be included. This effect can be easily included in the model by simply taking for the metamaterial the permittivity $\epsilon=\epsilon_{0}\left(-1+i \delta_{e}\right)$ and per- 

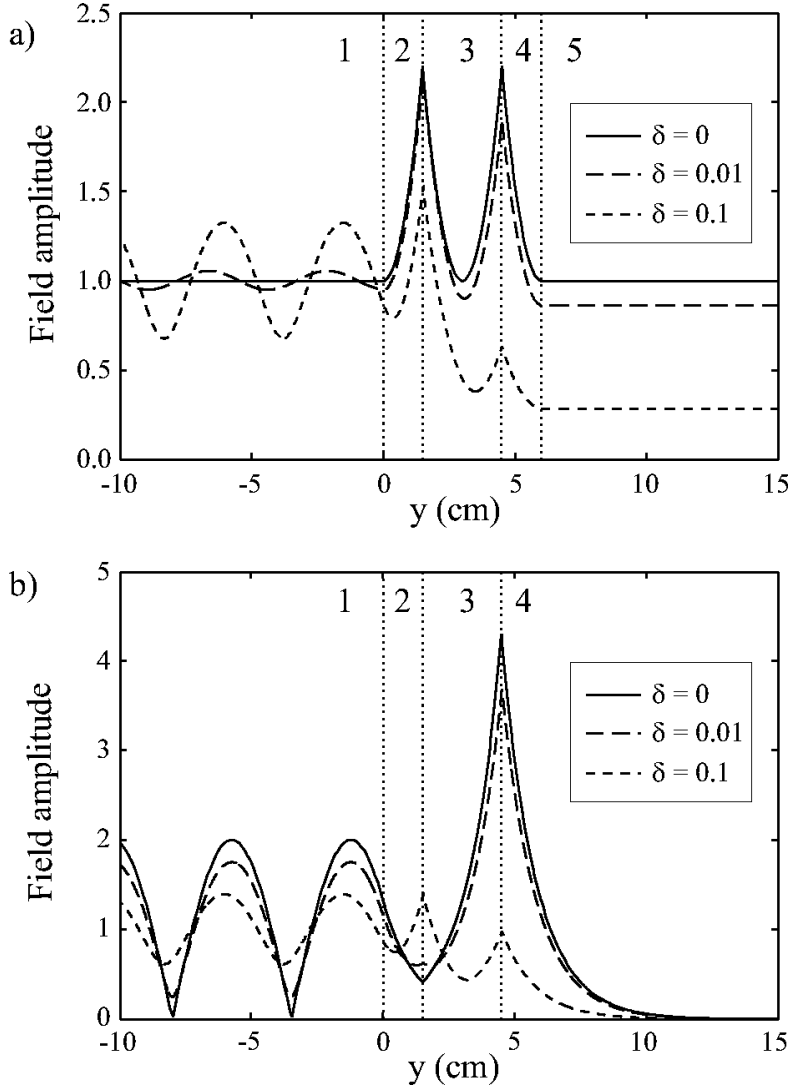

FIG. 3. (a) Effect of metamaterial losses on the field amplitude distribution inside the waveguide of Fig. 1. For simplicity, it has been imposed that magnetic and electric loss tangents are equal, so that $\epsilon=\epsilon_{0}(-1+i \delta)$ and $\mu=\mu_{0}(-1+i \delta)$. In (b), the last transition is eliminated $\left(l_{4} \rightarrow \infty\right)$.

meability $\mu=\mu_{0}\left(-1+i \delta_{m}\right)$, where $\delta_{e}$ and $\delta_{m}$ (electric and magnetic loss tangents) account for losses. Figure 3(a) shows the effect of losses on the field amplitude distribution, for different values of $\delta_{e}$ and $\delta_{m}$. The first and apparent conclusion is that the transmission decays when losses grow. However, in comparison with the tunneling through empty waveguides [dashed line in Fig. 2(a)], we can conclude that the transmission is still enhanced by more than one order of magnitude, even for loss tangents as high as 0.1. Therefore, although losses prevent the total transmission, the reported qualitative effect is still observed. Moreover, Fig. 3(b) shows that when the last transition is eliminated, the amplification of evanescent modes scheme is still clearly observed for loss tangents 0.01 . These results are qualitatively consistent with others shown in Ref. 11, which was obtained by finitedifference time-domain simulations for a metamaterial slab in free space.

Finally, the relevance of the length of the metamaterialfilled waveguide section, $l_{3}$, for the "perfect tunneling" effect is illustrated in Figs. 4 and 5. The transmittance degradation due to losses strongly depends on this length, specifically on the ratio between $l_{3}$ and the free space wavelength, $l_{3} / \lambda_{0}$ (see Fig. 4). It is very difficult to obtain near-perfect tunneling when the metamaterial section length is near to or higher than one wavelength. For instance, if $l_{3}=\lambda_{0}$, the loss tangent

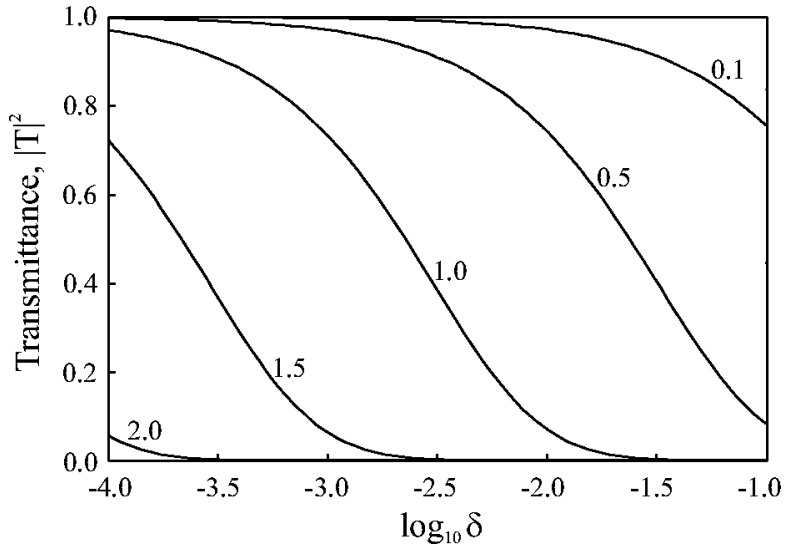

FIG. 4. Effect of the $l_{3} / \lambda_{0}$ ratio (numbers close to the lines) on the transmittance of the structure of Fig. 1 for different values of the loss tangent $\left(\delta_{m}=\delta_{e}=\delta\right.$ for simplicity). Waveguide parameters are $2 a / \lambda_{0}=0.8$ and $l_{2}+l_{4}=l_{3}$.

should be smaller than 0.001 to obtain a significant tunneling. However, if $l_{3}=0.1 \lambda_{0}$, a near-perfect tunneling is obtained even for moderate losses. These results are in agreement with previous ones reported for the related phenomenon of focusing. ${ }^{13}$ The ratio between the metamaterial filled section, $l_{3}$, and the wavelength is also crucial for the stability of the perfect tunneling effect. This influence is illustrated in Fig. 5, where the transmittance versus the ratio $\left(l_{2}+l_{4}\right) / l_{3}$ is shown for several $l_{3} / \lambda_{0}$ values. It is apparent from the figure that the perfect tunneling effect is much more stable when the metamaterial section is only a small fraction of the wavelength.

\section{B. Anisotropic metamaterial case}

There are other possibilities, different from the isotropic metamaterial filling, for the perfect tunneling in the device of Fig. 1. One of them is filling the waveguide section 3 with an indefinite magnetic medium with $\mu_{y y}=\mu_{z z}=\mu_{0}$ and $\mu_{x x}<0$. It follows from Maxwell equations that the attenuation constant and the wave impedance for the fundamental $T E_{10}$ mode of this waveguide section are given by ${ }^{12}$

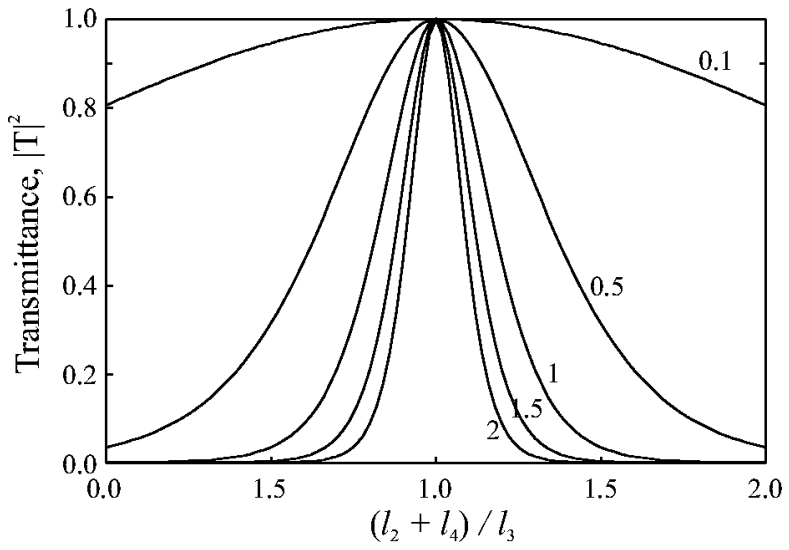

FIG. 5. Transmitted power through the device of Fig. 1 versus the ratio $\left(l_{2}+l_{4}\right) / l_{3}$ for several values of $l_{3} / \lambda_{0}$ (numbers close to the lines). Waveguide width is $a=0.8 \lambda_{0} / 2$. 
a)

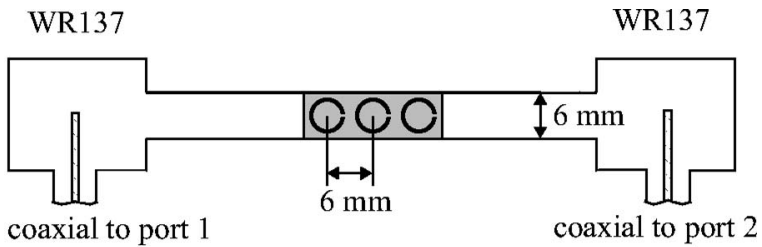

b)

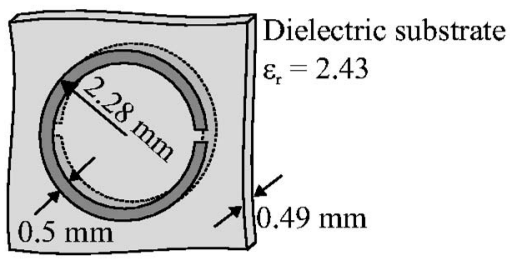

c)

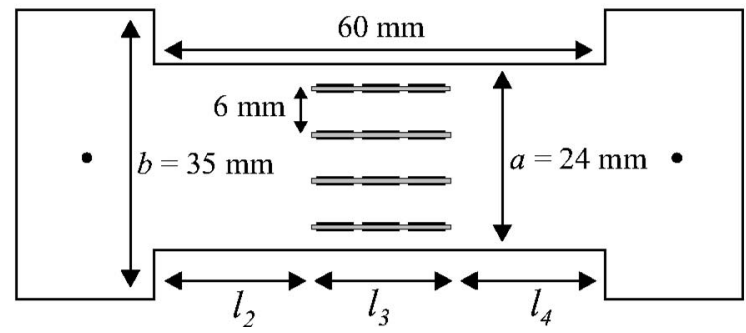

FIG. 6. Experimental setup, vertical cross section (a), detail of the BC-SRR (b), and horizontal cross section (c). The number of BC-SRRs in each arrow can vary between $m=0$ (empty waveguide) and $m=10$ (fully filled waveguide). The figure shows the specific case with $m=3$, where amplification and near-perfect tunneling are produced.

$$
\alpha_{3}^{2}=(\pi / a)^{2} \mu_{x x} / \mu_{0}-\omega^{2} \mu_{x x} \epsilon, \quad Z_{3}=-i \omega \mu_{x x} / \alpha_{3} .
$$

If $\mu_{x x}<0$, the first equation in (8) shows that propagation of electromagnetic waves along this waveguide is only possible if $\omega<\omega_{c}$, where $\omega_{c}=(\pi / a) / \sqrt{\epsilon \mu_{0}}$. On the contrary, if $\omega>\omega_{c}$ the waveguide is in cutoff and only evanescent modes can be excited. This amazing behavior, already reported in Ref. 12, simply reflects the anticutoff ${ }^{4}$ properties of the medium filling the waveguide section. If such medium has a dielectric permittivity $\epsilon>\epsilon_{0}$, it is possible to find a value for the waveguide cross section, $a$, for which the three waveguide sections 1,2 , and 3 are simultaneously in cutoff [the empty sections are in cutoff for any $\left.\omega<\omega_{c}^{0}=(\pi / a) / \sqrt{\epsilon_{0} \mu_{0}}\right]$. Thus, for an appropriate choice of $a, \mu_{x x}, \epsilon$, and $l_{i}$, the condition (6) can be fulfilled.

\section{EXPERIMENT AND SIMULATION}

\section{A. Experiment}

In order to check this theory, the experimental setup shown in Fig. 6 has been designed. A rectangular waveguide is placed between two commercial coaxial to rectangular waveguide transitions. The rectangular waveguide is filled by four rows of $m$ equally spaced broadside-coupled split ring resonators (BC-SRRs), which provide a resonant magnetic polarizability along the $x$ axis. ${ }^{14,15}$ These particles have well known magnetic properties and produce an artificial magnetic medium, with $\mu_{x x}<0$ in some frequency range between

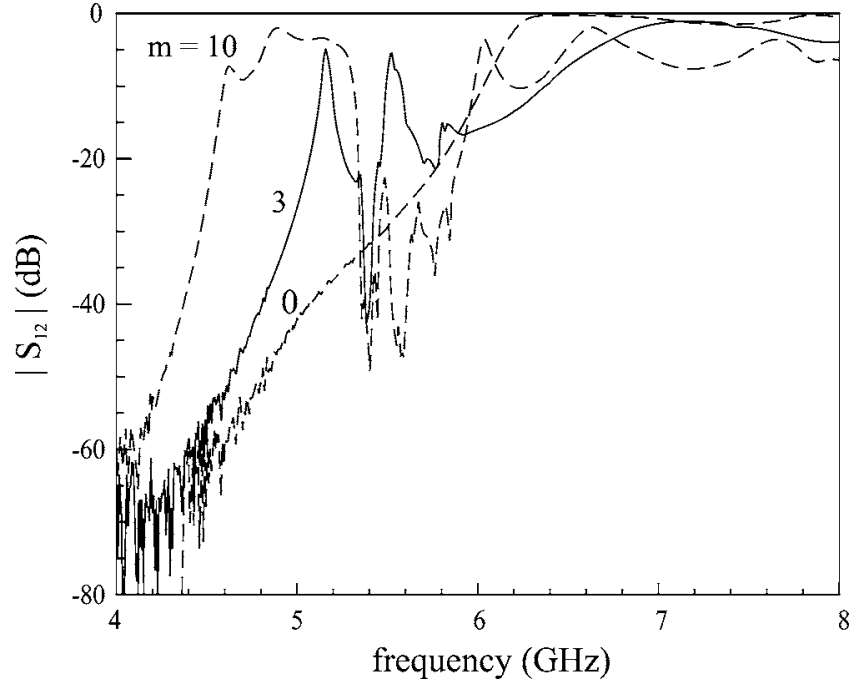

FIG. 7. Experimental values for the modulus of the transmission coefficient through the experimental setup of Fig. 6 for $m=0,10$ (dashed lines) and for $m=3$ (solid line).

5 and $6 \mathrm{GHz} .{ }^{16}$ Thus, the waveguide section at the center of Fig. 6 is filled by a metamaterial with a magnetic permeability along the $x$ axis, which is negative in this last frequency range. Besides, the dielectric permittivity of this medium should be slightly higher than that of vacuum, due to the nonresonant electric polarizability of the BC-SRRs. ${ }^{14}$

The experimental transmission coefficient for the cases of $m=0$ (empty waveguide), $m=10$ (fully filled waveguide), and $m=3$ (metamaterial filled waveguide section sandwiched between two empty sections) are shown in Fig. 7. The case $m=0$ simply shows the cutoff frequency of the empty waveguide, at $6.2 \mathrm{GHz}$. The case $m=10$ shows the first cutoff at $4.6 \mathrm{GHz}$, followed by a stop-band between 5.35 and 6.0 GHz. As it can be seen in the figure, inside this last frequency range the empty waveguide and the waveguide filled with the metamaterial are simultaneously in cutoff. Finally, a sharp increase of the transmission (of more than $20 \mathrm{dBs}$ over the empty waveguide) is observed at some frequency inside this last range when the metamaterial-filled waveguide is sandwiched between two empty waveguide sections (solid line in Fig. 7). We interpret these results as follows: as was already mentioned, the metamaterial (the BC-SRR medium) has an effective dielectric permittivity higher than those of vacuum. This effective permittivity explains the first cutoff frequency at $\omega_{c} / 2 \pi=4.6 \mathrm{GHz}$ for the waveguide filled by the metamaterial, well below the cutoff frequency of the empty waveguide (see the curves for $m=10$ and $m=0$ in Fig. 7). The stop band between 5.35 and $6.0 \mathrm{GHz}$ in the curve for $m=10$ (waveguide completely filled by the metamaterial) is due to the aforementioned frequency band of negative $\mu_{x x}$ in the metamaterial (note that in this frequency band we have simultaneously $\mu_{x x}<0$ and $\omega>\omega_{c}$ ). Inside this frequency band the empty and the metamaterial filled waveguides are both in cutoff. Thus, according to the previous theory, the transmission peak in the curve for $m=3$ should correspond to the tunneling by amplification of evanescent electromagnetic waves in the waveguide section loaded with the BC-SRRs-the section filled with the 


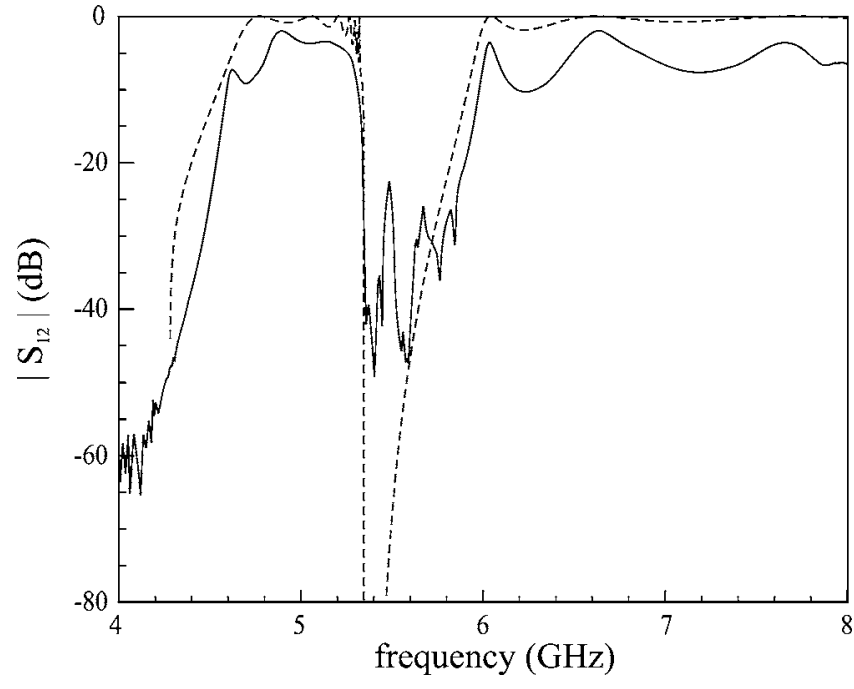

FIG. 8. Experimental (solid line) and calculated (dashed line) values for the modulus of the transmission coefficient through the experimental setup of Fig. 6 for $m=10$.

metamaterial-when it is sandwiched between two empty waveguide sections operating in cutoff (the specific case shown in Fig. 6).

\section{B. Comparison with theory}

The experimental results shown in Fig. 7 clearly suggest the presence of tunneling by amplification of evanescent waves in the BC-SRR filled waveguide section. However, they must be checked with the theoretical predictions before to reach a conclusion. In the following, the quantitative agreement between theory and experiment will be examined. The first step in this direction is the determination of the effective dielectric permittivity of the metamaterial from the first cutoff frequency of the BC-SRR filled waveguide. Since this cutoff frequency is given by $\omega_{c}=(\pi / a) / \sqrt{\epsilon_{\mathrm{eff}} \mu_{0}}$, a direct calculation leads to $\epsilon_{\mathrm{eff}} / \epsilon_{0}=1.844$. Then, a lossless DrudeLorentz model is assumed for the transverse permeability $\mu_{x x}$ of the metamaterial,

$$
\mu_{x x} / \mu_{0}=1+\frac{\delta f\left(2 f_{0}+\delta f\right)}{f_{0}^{2}-f^{2}},
$$

where $f$ is the frequency and the parameters $f_{0}$ and $\delta f$ stand for the frequency of resonance and the bandwidth of the region of negative $\mu_{x x}$. They have been determined by approximately adjusting the theoretical transmission to the experimental stop-band for $m=10$ (see Fig. 8). This procedure leads to the values $f_{0}=5.35 \mathrm{GHz}$ and $\delta f=0.55 \mathrm{GHz}$, that are consistent with previous theoretical and experimental results. ${ }^{16}$ By inserting these values in (3), the theoretical transmission coefficient for some structures with different values of $m$ has been calculated. Figure 9 shows these results for the $m=3,4,5$ cases (dashed lines). The transmission peaks at frequencies where the metamaterial filled waveguide section-the BC-SRR filled section-is not in cutoff $(f<5.35 \mathrm{GHz})$ can be interpreted as Fabry-Perot resonances in the metamaterial filled section of the waveguide, which
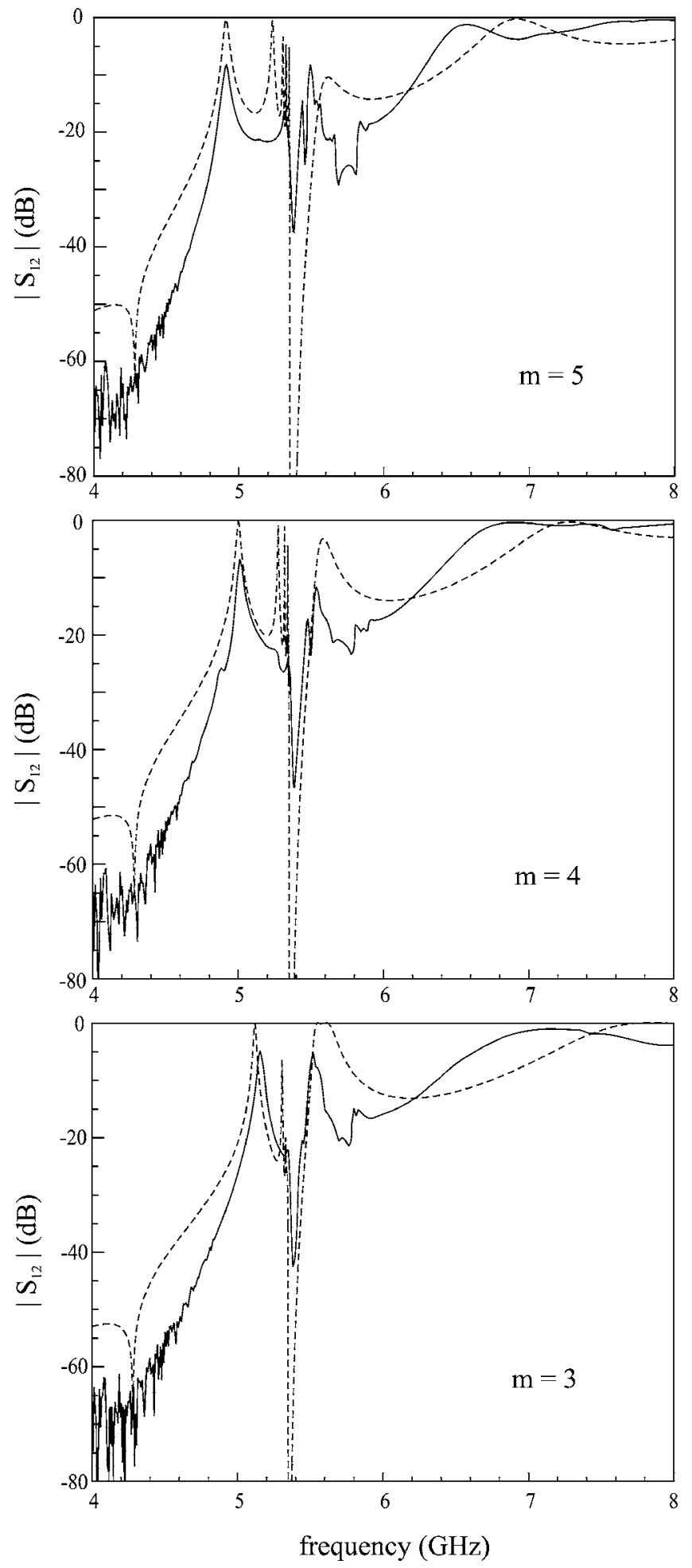

FIG. 9. Experimental (solid line) and calculated (dashed line) values for the modulus of the transmission coefficient through the experimental setup of Fig. 6 for $m=5,4,3$.

is not in cutoff. ${ }^{17}$ However, the peak inside the region $f_{0}<f<f_{0}+\delta f$ cannot be interpreted in this way, because the metamaterial filled waveguide section is in cutoff. This peak should correspond to the amplification condition (6). In fact, this peak is very near to the frequency where the theoretical values for the impedances of the hollow and the 


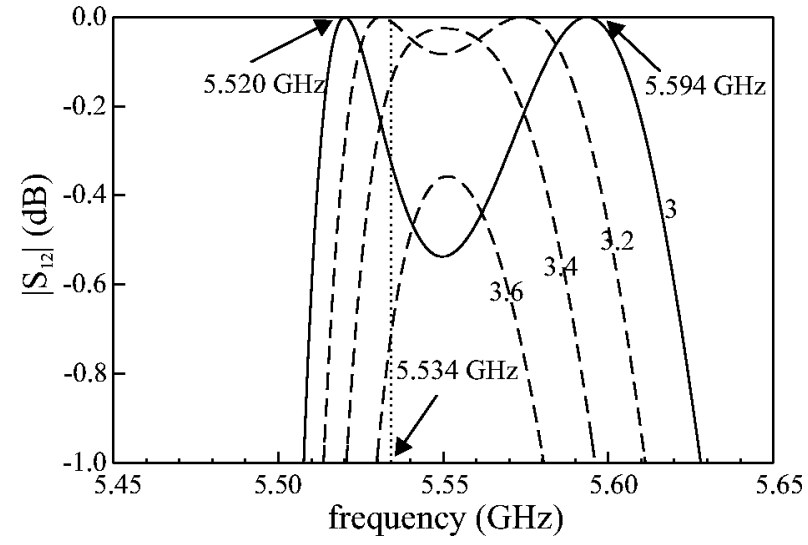

FIG. 10. "Double peak region" for the analyzed case, $m=3$ (zoom of Fig. 9), and some near cases. The numbers close to the curves are the length of the metamaterial section, $l_{3}$, measured in unit cells, $l_{3}=3 d, 3.2 d, \ldots, d$ being the distance between two nearest resonators $(d=6 \mathrm{~mm})$. At the frequency $5.534 \mathrm{GHz}$, the impedances $Z_{2}, Z_{3}$, and $Z_{4}$ are matched.

metamaterial filled waveguide [computed from (8)] are equal $\left(Z_{2}=Z_{3}=Z_{4}\right.$ at $\left.5.534 \mathrm{GHz}\right)$. For the considered structure, this frequency is very near to the theoretical frequency for which $\alpha_{2} l_{2}+\alpha_{3} l_{3}+\alpha_{4} l_{4}=0(5.498 \mathrm{GHz})$.

Up to now we have studied the theoretical curves. In the following we will check the agreement between theory and experiments. The experimental curves for the transmission coefficient are also included in Fig. 9 (solid lines). This figure shows a systematic coincidence in the location of the theoretical and the experimental peaks. Experimental curves-not shown here-for other values of $m$ in Fig. 6 (i.e., for other values of $l_{i}$ in Fig. 1) between $m=2$ and $m=9$ show a similar agreement with the theoretical results. This agreement proves the validity of the proposed theory. The coincidence between theory and experiments in the region $f_{0}<f<f_{0}+\delta f$ reveals, in addition, the presence of nearperfect tunneling due to the amplification of evanescent waves in the metamaterial. Quantitative differences between the theoretical and the experimental results can be due to three main reasons. First of all, ohmic losses in the rings strongly limit the amplification of evanescent waves in the

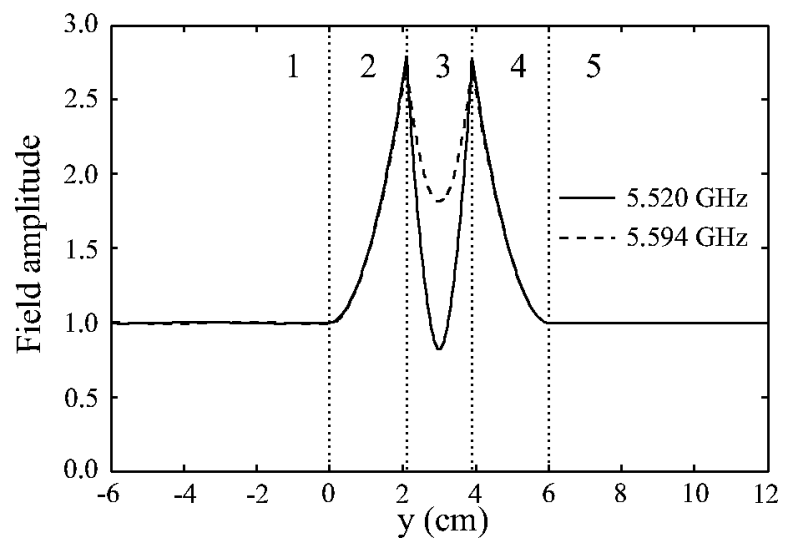

FIG. 11. Theoretical field amplitude inside the setup of Fig. 6 $(m=3)$ at the two perfect tunneling frequencies (see Fig. 10).

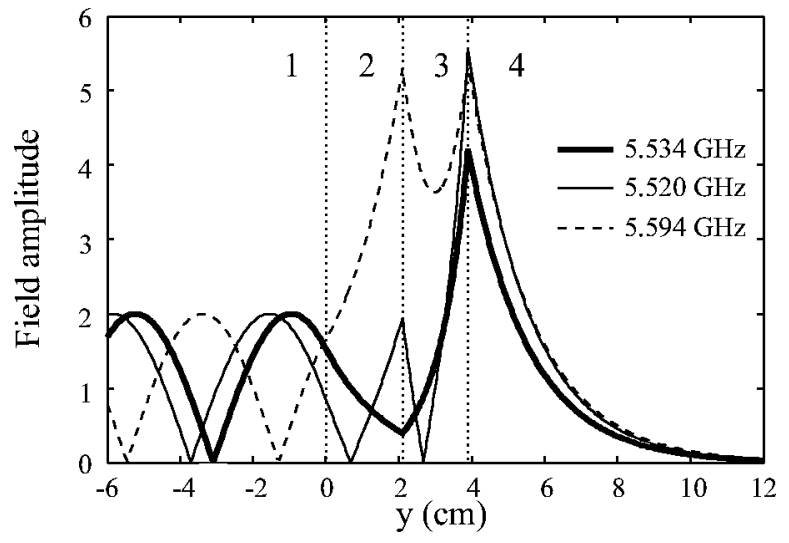

FIG. 12. Theoretical field amplitude inside a structure similar to the one shown in Fig. 6 for $m=3$, but now the last transition is eliminated $\left(l_{4} \rightarrow \infty\right)$.

metamaterial, ${ }^{4,18}$ so the amplitude of the peak is smaller than the predicted by the lossless theory. In addition, there is a mismatch at the input and the output of the experimental device, which only in a first approximation is accounted for by the impedance steps, as is assumed in (3). Finally, the limitations imposed to the assumed continuous medium approach by the nonvanishing size $(\sim \lambda / 10)$ of the unit cell should be also considered..$^{18}$ However, as it has been shown, these limitations do not completely suppress the effects predicted by the theory, making it possible to obtain near-perfect tunneling in a real experiments.

It was already mentioned that the frequency at which the theory predicts perfect matching $\left(Z_{2}=Z_{3}=Z_{4}\right.$ at $\left.5.534 \mathrm{GHz}\right)$ does not exactly coincide with the frequency at which $\alpha_{2} l_{2}+\alpha_{3} l_{3}+\alpha_{4} l_{4}=0(5.498 \mathrm{GHz})$. Since the values taken by $l_{i}$ in our practical devices are limited to an integer number of the unit cell $d=6 \mathrm{~mm}$ (i.e., $l_{3}=m \cdot d$ ), they can fulfill the perfect tunneling conditions (6) only approximately. Thus, a theoretical investigation on how the aforementioned frequency mismatch affects the analyzed phenomena is of interest. Actually, in the real experiment where $l_{3}=3 d$, our theory predicts a "double peak," at $5.520 \mathrm{GHz}$ and $5.594 \mathrm{GHz}$, around the impedance matching frequency, $5.534 \mathrm{GHz}$ (see Fig. 10). If a continuous variation of the length $l_{3}$ is assumed - the total length $l_{2}+l_{3}+l_{4}$ being constant- the theory predicts that these two peaks collapse to a single peak for some value of $l_{3}$ between $3.2 d$ and $3.4 d$. For higher values of $l_{3}$ perfect tunneling is not observed at any frequency. The theoretical distribution of the field amplitude inside the device for $l_{3}=3 d$ (which corresponds to the actual experiment with $m=3$ ) is represented in Fig. 11 at the frequencies of the two transmission peaks. Therefore, it seems that (6) is a sufficient but not a necessary condition for perfect tunneling. That is, perfect tunneling can be achieved even if the metamaterial section is not perfectly matched or if $\alpha_{2} l_{2}+\alpha_{3} l_{3}+\alpha_{4} l_{4}$ is not exactly zero, as is seen in Figs. 9 and 10.

In order to show the amplification of evanescent modes, in Fig. 12 we have represented the field amplitude inside the structure of Fig. 6 for $l_{4} \rightarrow \infty$ (no power transmission) at the different frequencies marked in Fig. 10. As expected, only when the perfect matching condition $\left(Z_{2}=Z_{3}=Z_{4}\right.$ at 


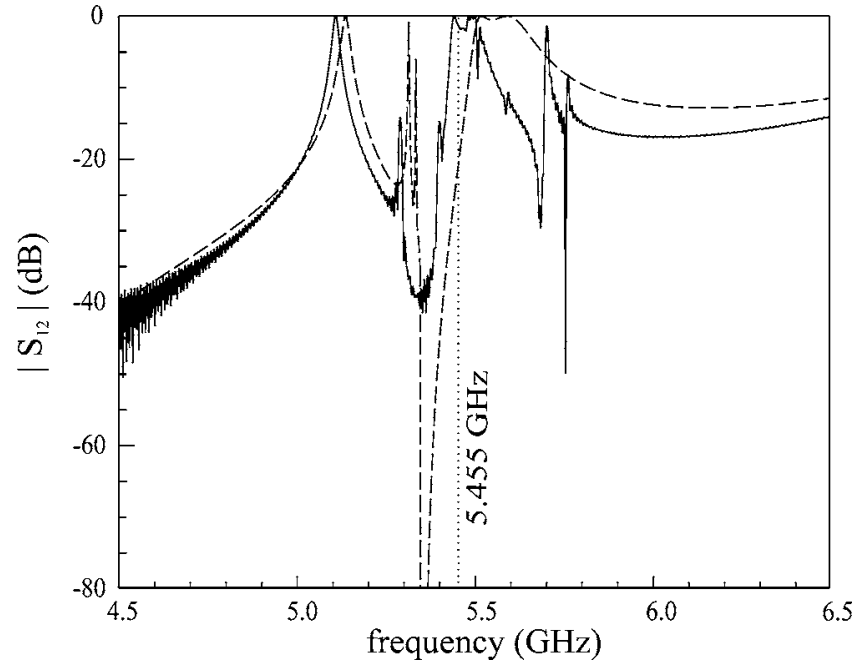

FIG. 13. Electromagnetic simulation results for the modulus of the transmission coefficient through the device of Fig. 6 for $m=3$ and without losses (solid line). The predictions of the proposed metamaterial effective medium theory are also shown (dashed lines).

$5.534 \mathrm{GHz})$ is fulfilled, this amplification of evanescent modes in the metamaterial filled waveguide is clearly observed in Fig. 12. At other frequencies, the excitation of reflected evanescent modes at the interfaces disguises this effect. In order to illustrate the above concepts, the time dependence of field amplitude has been shown in Ref. 19 for a monochromatic wave.

\section{Simulation}

In order to show graphically the reported amplification of evanescent modes in the BC-SRR loaded waveguide, we have carried out some electromagnetic simulations by using the commercial software CST Microwave Studio. First of all, the tunneling structure shown in Fig. 6 (with $m=3$ ) was simulated without losses. The results are shown in Fig. 13. The agreement between theory and simulations is apparent, except for a small frequency shift due to the limitations of the meshing procedure. To show the amplification of evanescent modes in the purely reactive configuration $\left(l_{4} \rightarrow \infty\right)$, the structure of Fig. 13 was simulated under the fundamental $T E_{10}$ mode incidence. The field distribution was computed at the frequency $f=5.455 \mathrm{GHz}$, between the transmission peaks of the simulation. The intensity of the magnetic field of the simulation in the middle plane of the waveguide is shown in Fig. 14. Except for the details of the SRR configuration, the decaying-amplifying - decaying sequence of the evanescent average waves in the in-cutoff waveguide sections can be clearly seen. It is worth noting that the above results are very sensitive to small changes in the frequency of operation. In fact, a change of a few tens of $\mathrm{MHz}$ is enough to strongly degrade the field patterns of Fig. 14. On the contrary, the perfect tunneling effect shown in Fig. 13 is much more stable
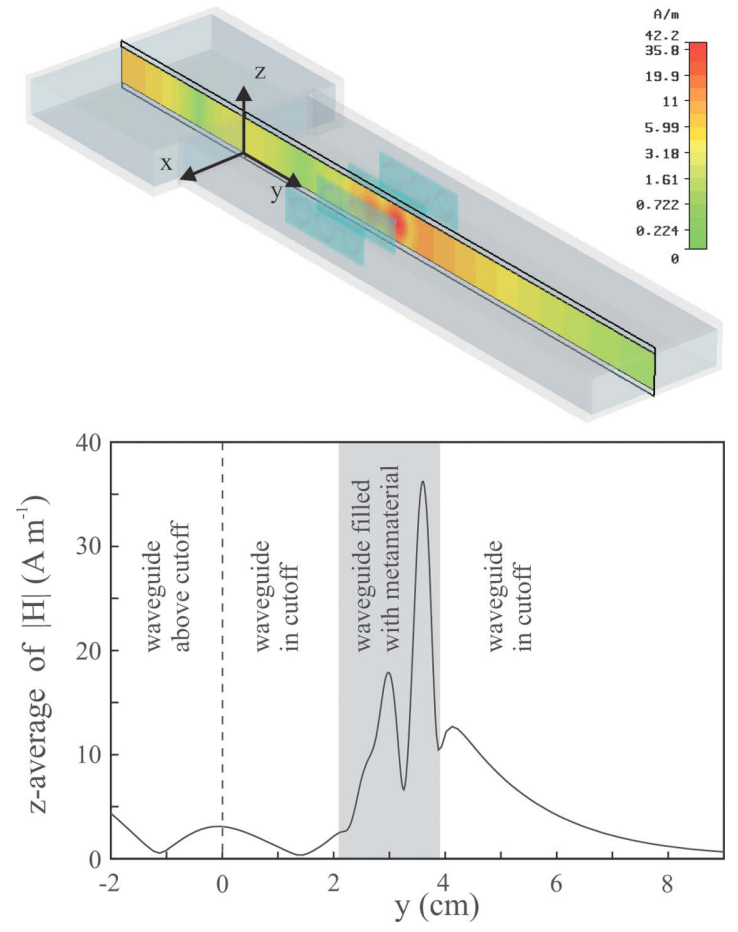

FIG. 14. (Color online) Intensity of the simulated magnetic field at the central plane of the waveguides, at $f=5.455 \mathrm{GHz}$. At this frequency the input waveguide is above cutoff, and the remaining waveguide sections operate in cutoff. The graphic shows the $z$-averaged intensity of the magnetic field.

with regard to these small variations in frequency. These results, once again, confirm the reported theory (see Fig. 12).

\section{CONCLUSIONS}

Enhanced tunneling (by several orders of magnitude) across a waveguide filled with a metamaterial and sandwiched between two empty sections operating in cutoff has been demonstrated. This effect has been explained by means of the effective medium metamaterial theory, according to which the decaying evanescent waves coming from the input are amplified in the metamaterial region (and, conversely, the backward decaying waves coming from the output, are also amplified in the backward direction inside the metamaterial). In particular, this effect has been experimentally verified when the metamaterial is an uniaxial anisotropic magnetic medium with a negative effective permeability along its optical axis. The experimental results show a very good qualitative and quantitative agreement with the theoretical predictions of the metamaterial theory, thus being an additional support for the validity of such theory.

\section{ACKNOWLEDGMENTS}

This work has been supported by the Spanish Ministry of Education and Science by project Contracts Nos. TIC20013163 and TEC2004-04249-C02-02). We also thank Professor Jan Zehetner from Czech Technical University for his kind collaboration. 
*Electronic address: juan_dbd@us.es

${ }^{1}$ V. G. Veselago, Sov. Phys. Usp. 10, 509 (1968).

${ }^{2}$ J. B. Pendry, Phys. Rev. Lett. 85, 3966 (2000).

${ }^{3}$ A. Alu and N. Engheta, IEEE Trans. Antennas Propag. 51, 2558 (2003).

${ }^{4}$ D. R. Smith and D. Schurig, Phys. Rev. Lett. 90, 077405 (2003).

${ }^{5}$ G. V. Eleftheriades, A. K. Iyer, and P. C. Kremer, IEEE Trans. Microwave Theory Tech. 50, 2702 (2002).

${ }^{6}$ A. Grbic and G. V. Eleftheriades, Phys. Rev. Lett. 92, 117403 (2004).

${ }^{7}$ S. Maslovski, S. Tretyakov, and P. Alitalo, J. Appl. Phys. 96, 1293 (2004).

${ }^{8}$ N. García and M. Nieto-Vesperinas, Phys. Rev. Lett. 88, 207403 (2002).

${ }^{9}$ R. E. Collin, Field Theory of Guided Waves, 2nd ed. (IEEE Press, New York, 1991).

${ }^{10}$ J. B. Pendry, Phys. Rev. Lett. 87, 249704 (2001).

${ }^{11}$ X. S. Rao and C. K. Ong, Phys. Rev. B 68, 113103 (2003).

${ }^{12}$ S. Hrabar and J. Bartolic, Proc. ICECOM 2003, Duborvnik (Croatia), 2003, p. 251.

${ }^{13}$ R. Marqués and J. Baena, Microwave Opt. Technol. Lett. 41, 290 (2004).
${ }^{14}$ R. Marqués, F. Medina, and R. Rafii-El-Idrissi, Phys. Rev. B 65, 144440 (2002).

${ }^{15}$ R. Marqués, J. Martel, F. Mesa, and F. Medina, IEEE Trans. Antennas Propag. 51, 2572 (2003).

${ }^{16}$ R. Marqués, J. Martel, F. Mesa, and F. Medina, Microwave Opt. Technol. Lett. 35, 405 (2002).

${ }^{17}$ In fact, at these peaks, the ratio between the length of the $\mathrm{BC}$ SRR filled waveguide, $l_{3}$, and the theoretical half-wavelength inside such waveguide, $\lambda_{g} / 2$ [computed from (9) and (8)] is approximately a constant $\left(2 l_{3} / \lambda_{g} \sim 0.73 \pm 0.01\right)$ for the three values of considered. This result evidences a Fabry-Perot-type resonance, with a complex reflection coefficient at the interface between the metamaterial filled waveguide and the hollow waveguides (note that, in this case, $Z_{3}$ is real whereas $Z_{2}=Z_{4}$ are imaginary).

${ }^{18}$ D. R. Smith, D. Schurig, M. Rosenbluth, S. Schultz, S. A. Ramakrishna, and J. B. Pendry, Appl. Phys. Lett. 82, 1506 (2003).

${ }^{19}$ See EPAPS Document No. E-PRBMDO-71-139519 containing two GIF movies. This document can be reached via a direct link in the online article's HTML reference section or via the EPAPS homepage (http://www.aip.org/pubservs/epaps.html). 